\title{
Besov spaces and Carleson measures on the ball
}

\author{
H. Turgay Kaptanoğlu ${ }^{1}$ \\ Department of Mathematics, Bilkent University, Ankara 06800, Turkey \\ Received 30 January 2006; accepted after revision 5 September 2006 \\ Available online 11 October 2006 \\ Presented by Paul Malliavin
}

\begin{abstract}
Carleson and vanishing Carleson measures for Besov spaces on the unit ball of $\mathbb{C}^{N}$ are defined using imbeddings into Lebesgue classes via radial derivatives. The measures, some of which are infinite, are characterized in terms of Berezin transforms and Bergman-metric balls, extending results for weighted Bergman spaces. Special cases pertain to Arveson and Dirichlet spaces, and a unified view with the Hardy-space Carleson measures are presented. Weak convergence in Besov spaces is characterized, and weakly 0-convergent families are exhibited. Carleson measures are applied to characterizations of functions in weighted Bloch and Lipschitz spaces. To cite this article: H.T. Kaptanoğlu, C. R. Acad. Sci. Paris, Ser. I 343 (2006).
\end{abstract}

(C) 2006 Académie des sciences. Published by Elsevier Masson SAS. All rights reserved.

\section{Résumé}

Les espaces de Besov et les mesures de Carleson dans la boule. Utilisant les inclusions dans les espaces de Lebesgue à l'aide des dérivées radiales nous définissons les mesures de Carleson et les mesures de Carleson évanescentes dans le cadre des espaces de Besov de la boule unité de $\mathbb{C}^{N}$. Ces mesures (certaines d'entre elles sont infinies) sont caractérisées à l'aide des transformées de Berezin et de boules dans la métrique de Bergman, ce qui nous permet d'étendre les résultats des espaces de Bergman avec poids. Notons les cas particuliers des espaces d'Arveson et de Dirichlet. Nous présentons un point de vue unifié avec les mesures de Carleson des espaces de Hardy. La convergence faible dans les espaces de Besov est caractérisée et nous donnons des exemples de familles qui convergent faiblement vers 0. Les mesures de Carleson sont utilisées pour caractériser les éléments des espaces de Bloch avec poids et des espaces de Lipschitz. Pour citer cet article : H.T. Kaptanoğlu, C. R. Acad. Sci. Paris, Ser. I 343 (2006). (c) 2006 Académie des sciences. Published by Elsevier Masson SAS. All rights reserved.

\section{Introduction}

Let $\mathbb{B}$ be the unit ball of $\mathbb{C}^{N}, H(\mathbb{B})$ the space of holomorphic functions on $\mathbb{B}$, and $v$ the volume measure on $\mathbb{B}$ normalized with $v(\mathbb{B})=1$. On $\mathbb{B}$, let also $\mathrm{d} v_{q}(z)=\left(1-|z|^{2}\right)^{q} \mathrm{~d} v(z)$, which are finite only for $q>-1$, where $|z|^{2}=\langle z, z\rangle$ and $\langle z, w\rangle=z_{1} \bar{w}_{1}+\cdots+z_{N} \bar{w}_{N}$. The corresponding Lebesgue classes are $L_{q}^{p}$. We denote by $b(w, r)$ the ball in the Bergman metric with center $w \in \mathbb{B}$ and radius $r \in(0, \infty)$.

\footnotetext{
E-mail address: kaptan@fen.bilkent.edu.tr (H.T. Kaptanoğlu).

URL: http://www.fen.bilkent.edu.tr/ kaptan/ (H.T. Kaptanoğlu).

1 The research of the author is partially supported by a Fulbright grant.
} 
For $s, t \in \mathbb{R}$, consider the linear transformations $I_{s}^{t}$ defined for $f \in H(\mathbb{B})$ by $I_{s}^{t} f(z)=\left(1-|z|^{2}\right)^{t} D_{s}^{t} f(z)$, where $D_{s}^{t}$ are the invertible radial differential operators on $H(\mathbb{B})$ of order $t$ for any $s$ defined as coefficient multipliers and discussed in $[8, \S 3]$. Every $I_{s}^{0}$ is the identity or inclusion.

Definition 1.1. Let $q \in \mathbb{R}$ and $0<p<\infty$. We define the Besov space $B_{q}^{p}$ to consist of all $f \in H(\mathbb{B})$ for which the function $I_{s}^{t} f$ belongs to $L_{q}^{p}$ for some $s, t$ satisfying

$$
q+p t>-1 \text {. }
$$

The $L_{q}^{p}$ norms of $I_{s}^{t} f$ are all equivalent; we call any one of them the $B_{q}^{p}$ norm of $f$ and denote it by $\|f\|_{B_{q}^{p}}$, where the term norm is used even when $0<p<1$.

Definition 1.1 is independent of $s, t$ under (1); see [8, Theorem 4.1] or [3, Theorem 5.12(i)].

When $q>-1, t=0$ satisfies (1) for any $p$, and the space $B_{q}^{p}$ coincides with the weighted Bergman space $A_{q}^{p}$. Thus Besov spaces generalize weighted Bergman spaces to all $q \in \mathbb{R}$. Moreover, $B_{-1}^{2}$ is the Hardy space $H^{2}, B_{-N}^{2}$ is the Arveson space, $B_{-(N+1)}^{2}$ is the Dirichlet space, and $B_{-(N+1)}^{p}$ are the Möbius-invariant Besov spaces. The explicit forms of the reproducing kernels $K_{q}(z, w)$ of $B_{q}^{2}$ for all $q \in \mathbb{R}$, the case $p=\infty$, various inclusions among $B_{q}^{p}$, and further background on them can be found in [3,8], and [7].

By Definition 1.1, $I_{s}^{t}$ with $s, t$ satisfying (1) is an imbedding of $B_{q}^{p}$ into $L_{q}^{p}$. But Carleson measures are often defined on Besov spaces with limited $q$ using inclusion as the imbedding into certain Lebesgue classes. However, descriptions of such Carleson measures for $q \leqslant-1$ are difficult to check, patchy, and not natural; see, for example, [10] for $q+p>-1$ and [1] for $q=-(N+1)$, both with $N=1$.

Our aim in this Note is to define Carleson measures for all Besov spaces and characterize them in a uniform manner. Detailed proofs and further results will be presented elsewhere.

\section{Carleson measures for Besov spaces}

Definition 2.1. We call a positive Borel measure $\mu$ on $\mathbb{B}$ a Carleson measure for $B_{q}^{p}$ provided some $I_{s}^{t}$ maps $B_{q}^{p}$ into $L^{p}(\mu)$ continuously.

Using derivatives to imbed holomorphic function spaces into Lebesgue classes is not uncommon; see [9] and its references, and [4], for example.

Theorem 2.2. Let $q \in \mathbb{R}$ be fixed but unrestricted. Let $p \in(0, \infty), r \in(0, \infty), s \in \mathbb{R}$ be given. The following conditions are equivalent for a positive Borel measure $\mu$ on $\mathbb{B}$.

(i) $\mu(b(w, r))=\mathcal{O}\left(v_{q}(b(w, r))\right)(w \in \mathbb{B})$.

(ii) The measure $\mu$ is a Carleson measure for $B_{q}^{p}$, that is, if t satisfies (1), then

$$
\int_{\mathbb{B}}\left|I_{s}^{t} f\right|^{p} \mathrm{~d} \mu=\mathcal{O}\left(\|f\|_{B_{q}^{p}}^{p}\right) \quad\left(f \in B_{q}^{p}\right) .
$$

(iii) If $t$ satisfies (1), then

$$
\left(1-|w|^{2}\right)^{N+1+q+p t} \int_{\mathbb{B}} \frac{\left(1-|z|^{2}\right)^{p t}}{|1-\langle z, w\rangle|^{(N+1+q+p t) 2}} \mathrm{~d} \mu(z)=\mathcal{O}(1) \quad(w \in \mathbb{B}) .
$$

It is clear from Theorem 2.2 that a Carleson measure $\mu$ is independent of $p, r, s, t$ as long as (1) holds. However, all conditions of Theorem 2.2 depend on $q$, and we call such a $\mu$ also a $q$-Carleson measure.

The left side of the equation in (iii) without the sup is a Berezin transform of $\mu$; cf. [6, §2.1].

Carleson measures need not be finite for $q \leqslant-1$, because $v_{q}$ is a Carleson measure for $B_{q}^{p}$ for any $p$, and $v_{q}(b(w, r))$ is of order $\left(1-|w|^{2}\right)^{N+1+q}$ for any given $r$. Hence, by (i), any finite Borel measure is a $q$-Carleson 
measure for $q \leqslant-(N+1)$. On the other hand, if $\mu$ is any $q$-Carleson measure, then (iii) implies that the measure $\left(1-|z|^{2}\right)^{p t} \mathrm{~d} \mu(z)$ is finite for any $p, t$ satisfying (1).

When $q>-1$, with $t=0$, Theorem 2.2 becomes a well-known result for weighted Bergman spaces; see [5, Theorem 2.36] for $N=1$. But it is possible to take $t \neq 0$ also with $q>-1$ as long as (1) holds; so Theorem 2.2 extends this known result also by giving equivalences with $I_{s}^{t}$ in place of inclusion.

\section{Vanishing Carleson measures}

Definition 3.1. We call a Carleson measure for $B_{q}^{p}$ a vanishing Carleson measure for $B_{q}^{p}$ whenever some $I_{s}^{t}$ mapping $B_{q}^{p}$ into $L^{p}(\mu)$ is further compact.

Theorem 3.2. The little "oh" version as $|w| \rightarrow 1$ of Theorem 2.2 holds for vanishing Carleson measures.

The next result is essential for the proof of Theorem 3.2. A similar result holds for composition operators on similar spaces too; see [5, Proposition 3.11].

Theorem 3.3. Let $\mu$ be positive Borel measure on $\mathbb{B}$ and $s, t$ satisfy (1). The operator $I_{s}^{t}: B_{q}^{p} \rightarrow L^{p}(\mu)$ is compact if and only if for any sequence $\left\{f_{k}\right\}$ in $B_{q}^{p}$ with $\left\{\left\|f_{k}\right\|_{B_{q}^{p}}\right\}$ bounded and $\left\{I_{s}^{t} f_{k}\right\}$ converging to 0 uniformly on compact subsets of $\mathbb{B}$, we have $\left\|I_{s}^{t} f_{k}\right\|_{L^{p}(\mu)} \rightarrow 0$.

Theorem 3.4. For $1<p<\infty$, a sequence $\left\{f_{k}\right\}$ converges to 0 weakly in $B_{q}^{p}$ if and only if $\left\{\left\|f_{k}\right\|_{B_{q}^{p}}\right\}$ is bounded and $\left\{I_{s}^{t} f_{k}\right\}$ converges to 0 uniformly on compact subsets of $\mathbb{B}$ for some $s, t$ satisfying (1) and

$$
q+1<p(s+1) .
$$

Condition (2) is necessary and sufficient for the existence of extended Bergman projections from $L_{q}^{p}$ onto $B_{q}^{p}$ for $p \geqslant 1$; see [8, Theorem 1.2].

It is well known $([12, \S 6.1])$ that if $q>-1$, then suitable powers of normalized reproducing kernels converge to 0 weakly as $|w| \rightarrow 1$ in the Bergman spaces $A_{q}^{p}$ with $p>1$. In Besov spaces $B_{q}^{p}$ with $-(N+1)<q \leqslant-1$ when the associated reproducing kernels are binomial, the same idea works too. But it cannot work in $B_{q}^{p}$ with $q<-(N+1)$ when the associated reproducing kernels are bounded.

Example 1. Fix $q$ and let $t$ satisfy (1). Pick $c_{k} \in \mathbb{C}$ such that $c_{k}$ is of order $k^{(N+1+q+p t) 2 / p-1-t}$ as $k \rightarrow \infty$, and put $f_{w}(z)=\sum_{k=0}^{\infty} c_{k}\langle z, w\rangle^{k}$. Set $g_{w}(z)=f_{w}(z) /\left\|f_{w}\right\|_{B_{q}^{p}}$. Then $\left\|g_{w}\right\|_{B_{q}^{p}}=1$ and $I_{s}^{t} g_{w} \rightarrow 0$ uniformly as $|w| \rightarrow 1$ on compact subsets of $\mathbb{B}$.

Example 2. Consider the case of a Hilbert space, $p=2$, in Example 1. Let $s$ satisfy (2) and let $t=-q+s$, which satisfies (1). Then we can take $f_{w}(z)=K_{s}(z, w)$ and $g_{w}(z)=K_{s}(z, w) /\left\|K_{s}(\cdot, w)\right\|_{B_{q}^{2}} \in B_{q}^{2}$. It turns out that $f_{w}$ is the kernel for the evaluation of the derivative $D_{s}^{-q+s} f$ of $f \in B_{q}^{2}$ at $w \in \mathbb{B}$. A similar weak convergence result can be found in [5, Proposition 7.13].

The results in this section with $q>-1$ and $t \neq 0$ are new for weighted Bergman spaces too.

\section{Hardy spaces}

Consider $B_{-1}^{2}=H^{2}$. Now (1) requires a $t>0$, no matter how small. So Definition 2.1 is about Carleson measures different from the usual Carleson measures on $H^{2}$. However, replace $\|f\|_{B_{-1}^{2}}$ on the right side of the equation in Theorem 2.2(ii) by $\|f\|_{B_{-1}^{2}} \sqrt{(2 t)(2 t+1) \cdots(2 t+N-1) / N !}$, which is an equivalent norm for $B_{-1}^{2}$. As $t \rightarrow 0^{+}$, it tends to the usual norm on $H^{2}$, and Theorem 2.2(iii) becomes equivalent to being a usual Carleson measure on $H^{2}$, hence on $H^{p}$; see [12, Corollary 8.2.3] for $N=1$. We thereby unify the theory of Carleson measures on weighted Bergman, Besov, and Hardy spaces simultaneously. 


\section{Weighted Bloch and Lipschitz spaces}

Definition 5.1. For any $\alpha \in \mathbb{R}$, we define the weighted Bloch space $\mathcal{B}^{\alpha}$ to consist of all $f \in H(\mathbb{B})$ for which $\left(1-|z|^{2}\right)^{\alpha} I_{s}^{t} f(z)$ is bounded on $\mathbb{B}$ for some $s, t$ satisfying $\alpha+t>0$. The weighted little Bloch space $\mathcal{B}_{0}^{\alpha}$ is that subspace of $\mathcal{B}^{\alpha}$ consisting of those $f$ for which $\left(1-|z|^{2}\right)^{\alpha} I_{s}^{t} f(z)$ vanishes on the boundary of $\mathbb{B}$ for some $s, t$ satisfying $\alpha+t>0$.

Independence of such $s, t$ is similar to that for Besov spaces. The spaces $\mathcal{B}^{0}$ and $\mathcal{B}_{0}^{0}$ are the classical Bloch and little Bloch spaces. By [13, Theorems 7.17 and 7.18], the spaces $\mathcal{B}^{\alpha}$ and $\mathcal{B}_{0}^{\alpha}$ for $\alpha<0$ are the holomorphic Lipschitz spaces $\Lambda_{-\alpha}$ and $\Lambda_{-\alpha, 0}$ of the ball.

Theorem 5.2. Given $\alpha, q, p, s$, let $t$ satisfy (1), and put $u=(N+1+q-\alpha p+p t) / p$. A function $h \in H$ (B⿺ lies in $\mathcal{B}^{\alpha}$ if and only if $\mathrm{d} \mu=\left|I_{s}^{u} h\right|^{p} \mathrm{~d} v_{q+\alpha p}$ is a $q$-Carleson measure. Also $h$ lies in $\mathcal{B}_{0}^{\alpha}$ if and only if $\mu$ is a vanishing $q$-Carleson measure.

The $u$ given in the statement satisfies $\alpha+u>0$, and the only-if parts work for any such $u$.

Certain limited ( $p=2$ and others) special cases of Theorem 5.2 for the classical Bloch spaces $(\alpha=0)$ are contained in [2] and [11], among others.

\section{Acknowledgements}

Parts of this work were done at Middle East Technical University and the University of Virginia. The author thanks the former for granting a sabbatical leave, the Department of Mathematics of the latter, the operator theory group there, and especially Thomas Kriete and Barbara MacCluer, for their hospitality.

\section{References}

[1] J. Arcozzi, R. Rochberg, E. Sawyer, Carleson measures for analytic Besov spaces, Rev. Mat. Iberoamericana 43 (1991) $443-510$.

[2] R. Aulaskari, D.A. Stegenga, J. Xiao, Some subclasses of BMOA and their characterization in terms of Carleson measures, Rocky Mountain J. Math. 26 (1996) 485-506.

[3] F. Beatrous, J. Burbea, Holomorphic Sobolev spaces on the ball, Dissertationes Math. 276 (1986) 57 pp.

[4] B.R. Choe, H. Koo, H. Yi, Carleson type conditions and weighted inequalities for harmonic functions, Osaka J. Math. 39 (2002) $945-962$.

[5] C. Cowen, B.D. MacCluer, Composition Operators on Spaces of Analytic Functions, CRC, Boca Raton, 1995.

[6] H. Hedenmalm, B. Korenblum, K. Zhu, Theory of Bergman Spaces, Springer, New York, 2000.

[7] H.T. Kaptanoğlu, Besov spaces and Bergman projections on the ball, C. R. Math. Acad. Sci. Paris, Ser. I 335 (2002) $729-732$.

[8] H.T. Kaptanoğlu, Bergman projections on Besov spaces on balls, Illinois J. Math. 49 (2005) 385-403.

[9] D.H. Luecking, Embedding theorems for spaces of analytic functions via Khinchine's inequality, Michigan Math. J. 40 (1993) $333-358$.

[10] Z. Wu, Carleson measures and multipliers for Dirichlet spaces, J. Funct. Anal. 169 (1999) 148-163.

[11] W. Yang, Carleson type measure characterization of $Q_{p}$ spaces, Analysis 18 (1998) 345-349.

[12] K. Zhu, Operator Theory in Function Spaces, Dekker, New York, 1990.

[13] K. Zhu, Spaces of Holomorphic Functions in the Unit Ball, Springer, New York, 2005. 